\title{
Preparation and evaluation of mesoporous cellular foams coating of solid-phase microextraction fibers by determination of tetrabromobisphenol A, tetrabromobisphenol S and related compounds
}

\author{
Xuemei Wang a,b ${ }^{\mathrm{a}}$, Jiyan Liu ${ }^{\mathrm{a}}$, Aifeng Liu ${ }^{\mathrm{a}}$, Qian Liu ${ }^{\mathrm{a}}$, Xinzhen Du ${ }^{\mathrm{b}}$, Guibin Jiang ${ }^{\mathrm{a}, *}$ \\ a State Key Laboratory of Environmental Chemistry and Ecotoxicology, Research Center for Eco-Environmental Sciences, Chinese Academy of Sciences, Beijing 100085, PR China \\ ${ }^{\mathrm{b}}$ College of Chemistry and Chemical Engineering, Northwest Normal University, Lanzhou 730070, PR China
}

\section{H I G H L I G H T S}

- Two novel mesoporous cellular foams were synthesized and used as SPME coating.

- Four SPME fibers were prepared by sol-gel technology and immobilized resin method.

- New coating had excellent extraction ability to seven brominated flame retardants.

\section{A R T I C L E I N F O}

\section{Article history:}

Received 3 August 2012

Received in revised form

15 September 2012

Accepted 19 September 2012

Available online 25 September 2012

\section{Keywords:}

Solid-phase microextraction

Fiber coating

Mesoporous cellular foams material

Brominated flame retardants

\section{G R A P H I C A L A B S T R A C T}
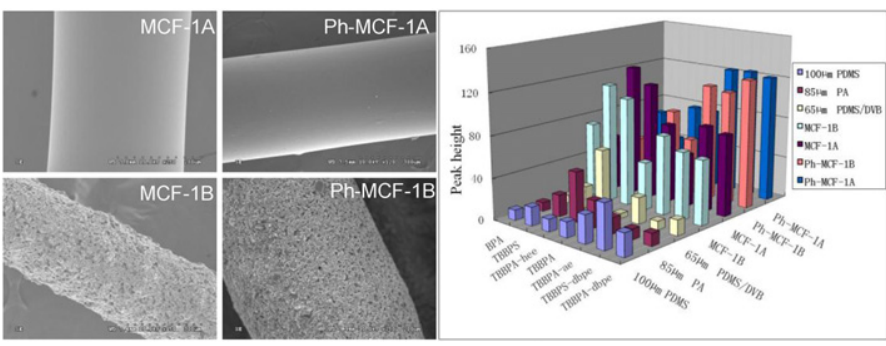

\begin{abstract}
A B S T R A C T
Two kinds of mesoporous cellular foams (MCFs), including mesoporous silica materials (MCF-1) and phenyl modified mesoporous materials (Ph-MCF-1), were synthesized and for the first time used as fibercoating materials for solid-phase microextraction (SPME). By using stainless steel wire as the supporting core, four types of fibers were prepared by sol-gel method and immobilized by epoxy-resin method. To evaluate the performance of the home-made fibers for SPME, seven brominated flame retardants (BFRs), including tetrabromobisphenol A (TBBPA), tetrabromobisphenol S (TBBPS) and related compounds were selected as analytes. The main parameters that affect the extraction and desorption efficiencies, such as extraction temperature, extraction time, desorption time, stirring rate and ionic strength of samples were investigated and optimized. The optimized SPME coupled with high performance liquid chromatography (HPLC) was successfully applied to the determination of the seven BFRs in water samples. The linearity range was from 5.0 to $1000 \mu \mathrm{g} \mathrm{L}^{-1}$ for each compound except TBBPS (from 1.0 to $1000 \mu \mathrm{g} \mathrm{L}^{-1}$ ), with the correlation coefficients $\left(r^{2}\right)$ ranging from 0.9993 to 0.9999 . The limits of detection of the method were $0.4-0.9 \mu \mathrm{g} \mathrm{L}^{-1}$. The relative standard deviations varied from 1.2 to $5.1 \%(n=5)$. The repeatability of fiber-to-fiber and batch-to-batch was $2.5-6.5 \%$ and 3.2-6.7\%. The recoveries of the BFRs from aqueous samples were in the range between 86.5 and $103.6 \%$. Compared with three commercial fibers $(100 \mu \mathrm{m}$ PDMS, $85 \mu \mathrm{m}$ PA and $65 \mu \mathrm{m}$ PDMS/DVB), the MCFs-coated fiber showed about 3.5-fold higher extraction efficiency.
\end{abstract}

(C) 2012 Elsevier B.V. All rights reserved.

\footnotetext{
* Corresponding author. Tel.: +86 10 62849334; fax: +861062839179.

E-mail address: gbjiang@rcees.ac.cn (G. Jiang).
}

\section{Introduction}

Sample preparation is one of the most crucial and important steps in the process of analysis, by some estimates [1], about $60 \%$ of the work time and operating cost is spent on preparing samples before introducing them into analytical instruments. It is often the bottleneck for obtaining a desired analytical result, especially for 
the determination of trace analytes in a complex matrix sample. As an efficient and remarkable sample preparation technique, solidphase microextraction (SPME) has attracted increasing attention due to its high efficiency, simplicity, solvent-free and facilitation in automation and coupling techniques [2-4]. In general, the fiber coating is considered to be the key factor in SPME technique [5-7]. Till now, the varieties of commercially available SPME fibers are increasing. Many researchers focus on developing new long lifetime, low cost and more stable SPME fibers and coatings with higher extraction efficiency, better reproducibility and higher selectivity by using new preparation methods or new materials.

In the past years, various SPME coating approaches such as electrochemical procedures [8,9], immobilized resin [10,11], sol-gel technology [12,13] and molecular imprinted technology [6,14] have been developed. For new coating materials, high-porosity materials have attracted much attention recently because of their large specific surface areas and high extraction capacities resulting in significant improvement in sensitivity, such as carbon nanotubes [15], graphene [16,17] and metal-organic framework [7]. Mesoporous materials are considered the high-efficiency sorbents due to high specific surface area, controllable pore size, narrow pore size distribution, thermal and mechanical stability, which have various applications in chemical industry [18,19], environment [10,18-20], medicine [21,22], adsorption and separation [23]. Compared with conventional mesoporous materials, mesoporous cellular foams (MCFs) have aerogel like, three-dimensional, continuous and ultralarge-pore structure through incorporation of swelling agent [9]. Moreover, MCFs exhibit much higher catalytic activity due to their fast mass transfer kinetics, good accessibility for large molecules (e.g. polymers, enzymes) and their large pore volumes are beneficial in various processes $[24,25]$. These properties make MCFs as ideal candidate for adsorption and separation, especially in sample pretreatment. However, to the best of our knowledge, there is no report on application of MCFs as SPME fiber coating.

Herein, two types of MCFs materials, including MCF-1 and PhMCF-1 (phenyl modified MCF-1), were synthesized and used as coating materials for preparing SPME fibers, based on two types of immobilized methods by sol-gel technology and by epoxyresin. In order to evaluate the extraction performance and stability of the prepared fibers, seven brominated flame retardants (BFRs) were selected as analytes. BFRs have been widely used and caused increasing concern on environmental and human health due to the potential endocrine disruptors [26-28]. Tetrabromobisphenol A (TBBPA) was the most used (59\%) product because of the ban of polybrominated diphenyl ethers (PBDEs) [29,30]. Tetrabromobisphenol S (TBBPS) is another type of BFRs like TBBPA which are widely used in Asia. At present, there are few papers about the sample preparation of TBBPA, TBBPS and related compounds. In this study, the home-made SPME fibers were applied to the simultaneous extraction of TBBPA, TBBPS and related compounds in real water samples.

\section{Experimental}

\subsection{Materials and chemicals}

Stainless steel wires (O.D., $0.15 \mathrm{~mm}$ ) were purchased from AnTing Micro-Injector Factory (Shanghai, China); commercial manual sampling SPME devices, fiber holder and commercial fibers (100 $\mu \mathrm{m}$ PDMS, $85 \mu \mathrm{m}$ PA and $65 \mu \mathrm{m}$ PDMS/DVB) were obtained from Supelco (Bellefonte, PA, USA).

Phenyltrimethoxysilane (PTMS) (97\%) and poly(ethylene glycol)-block-poly(propylene glycol)-block-poly(ethylene glycol) $\left(\mathrm{EO}_{80} \mathrm{PO}_{30} \mathrm{EO}_{80}\right.$, Pluronic $\left.\mathrm{F} 68, \mathrm{Ma}=8400\right)$ were purchased from
Sigma-Aldrich (St Louis, MO, USA). Poly(methylhydrosiloxane) (PMHS) and hydroxylterminated silicone oil (OH-TSO) were purchased from Alfa Aesar (Ward Hill, MA, USA). HPLC-grade acetonitrile, tetraethyl orthosilicate (TEOS) (98\%) and trifluoroacetic acid (TFA) (99.5\%) were bought from J.T. Baker (Phillipsburg, NJ, USA). Cyclohexane (CHE, 99\%), hydrochloric acid ( $\mathrm{HCl}, 37 \%)$, ethanol (EtOH, 95\%), and epoxy resin were obtained from Beijing Chemical Works (Beijing, China). These reagents were analytical grade and used as purchased without further purification.

Bisphenol A (BPA), TBBPA, tetrabromobisphenol-A-bis(allyl ether) (TBBPA-ae), tetrabromobisphenol-A-bis(2,3-dibromopropyl ether) (TBBPA-dbpe), and TBBP-A bis(2-hydroxyethyl ether) (TBBPA-hee) were obtained from Sigma-Aldrich (St Louis, MO, USA). TBBPS and tetrabromobisphenol-S-bis(2,3-dibromopropyl ether) (TBBPS-dbpe) were from Beijing APIS Biotechnology Ltd. (Beijing, China). Physical-chemical properties of TBBPA, TBBPS and their related compounds were listed in Supplementary data 1 . The stock standard solution of $100 \mathrm{mg} \mathrm{L}^{-1}$ of each compound was prepared in methanol and stored in amber bottles in the refrigerator at $4{ }^{\circ} \mathrm{C}$. The standard working solutions were prepared by diluting the stock standard solution with methanol to the required concentration, as required. Ultrapure water was obtained from Milli-Q (Advantage A10) water purification system.

A river water sample was collected from Xiaoqing River and treated wastewater was from a plant which produce TBBPA and other brominated flame retardants (Shandong Province, China). All these samples were filtrated through $0.45 \mu \mathrm{m}$ glass fiber membrane (Automatic Science, China) and stored in brown glass bottles at $4{ }^{\circ} \mathrm{C}$.

\subsection{Apparatus and instrumentation}

FT-IR spectra were recorded on a Digilab FTS3000 FT-IR spectrometer using the $\mathrm{KBr}$ wafer technique. Scanning electron microscope (SEM) images were recorded on S-3000N, to visualize the morphology and size distribution of the materials. Transmission electron microscope (TEM) was operated on HITACHI-7500 with an accelerating voltage of $80 \mathrm{kV}$. The small-angle X-ray diffraction (SAXRD) measurements were carried out on a X'Pert PRO MPD powder diffractometer, using $\mathrm{CuK} \alpha$ radiation $(40 \mathrm{kV}, 30 \mathrm{~mA})$ source with a resolution of $0.02^{\circ}$ and scanning speed of $0.5^{\circ} \mathrm{min}^{-1}$. Nitrogen adsorption-desorption isotherms were performed at $-196^{\circ} \mathrm{C}$ on a Micromeritics ASAP 2000 analyzer.

The Agilent 1100 series HPLC-UV system (Agilent Technologies, USA) with a variable wavelength detector and a reverse phase $C_{18}$ HPLC column (Acclaim120, $5 \mu \mathrm{m}, 150 \mathrm{~mm} \times 4.6 \mathrm{~mm}$, Dionex) were used for quantitative analysis. The mobile phase at a flow rate of $1 \mathrm{~mL} \mathrm{~min}^{-1}$ was mixture of water (component A) and acetonitrile (component $\mathrm{B}$ ) with gradient elution of $0-3.0 \mathrm{~min} 20 \% \mathrm{~A}, 80 \% \mathrm{~B}$; 3.0-10.5 min $0 \%$ A, $100 \%$ B; 10.5 min stop. The wavelength of UV detector was set at $214 \mathrm{~nm}$. The SPME-HPLC interface (Cat. No. 57353) is a commercially available desorption chamber and purchased from Supelco; it is sealed by PEEK (polyether ether ketone) septum which is tight enough to withstand pressure as high as 29.7 MPa.

\subsection{Synthesis of MCF-1 and Ph-MCF-1}

The synthesis of MCF-1 was as follows: $1.45 \mathrm{~g}$ of F68 was dissolved in $10 \mathrm{~mL}$ of mixture of $\mathrm{HCl}(37 \%)$ and $65 \mathrm{~g}$ of deionized $\mathrm{H}_{2} \mathrm{O}$ with vigorous stirring at $40^{\circ} \mathrm{C}$ for $1 \mathrm{~h}$. Then $0.5 \mathrm{~g}$ of $\mathrm{CHE}$ was added dropwise into the mixture under vigorous stirring. After $1 \mathrm{~h}$, $2.4 \mathrm{~mL}$ of TEOS was added dropwise and the solution was continuously stirred for $24 \mathrm{~h}$ at $40^{\circ} \mathrm{C}$. The mixture was transferred into Teflon-lined autoclaves, heated at $100^{\circ} \mathrm{C}$ and allowed to stand for overnight. The solid precipitate was recovered by filtration, washed 
several times with distilled water, and dried at $80^{\circ} \mathrm{C}$ in a vacuum drier for $3 \mathrm{~h}$. The organic template was removed by heating at a rate of $5^{\circ} \mathrm{C} \mathrm{min}^{-1}$ up to $500^{\circ} \mathrm{C}$ and then held for $5 \mathrm{~h}$ in air.

The synthesis of Ph-MCF-1 was improved based on our previous report [31]. Briefly, it is similar to synthesis method of Ph-SBA15 with using F68 as templating agent and CHE as swelling agent, which is an environmentally friendly and easily available cheap solvent.

\subsection{Preparation of SPME fibers}

Prior to coating, the stainless steel wire (length $5 \mathrm{~cm}$ ) were chemically etched for $30 \mathrm{~min}$ with hydrochloric acid to increase roughness and activate the auto-protective hydroxyl layer of the stainless steel surface. Afterwards, the wires were rinsed with deionized water and air dried in an oven at $100^{\circ} \mathrm{C}$ for $30 \mathrm{~min}$. Two approaches were used for coating fibers, including sol-gel and immobilizing with epoxy-resin.

The sol-gel solution was prepared as follows: $90 \mathrm{mg}$ of MCF1 (or Ph-MCF-1) was dissolved in $250 \mu \mathrm{L}$ of dichloromethane and then $250 \mu \mathrm{L}$ of TEOS, $50 \mu \mathrm{L}$ of OH-TSO, and $30 \mu \mathrm{L}$ of PMHS were added. The solution was mixed thoroughly for $20 \mathrm{~min}$ by ultrasonic agitation. $200 \mu \mathrm{L}$ of TFA ( $5 \%$ water) was subsequently added to the resulting solution with ultrasonic agitation for another $5 \mathrm{~min}$. The mixture was then centrifuged at $5000 \mathrm{rpm}$ for $5 \mathrm{~min}$, and the top clear sol solution was used for fiber coating.

For the fibers prepared with sol-gel method, the treated wire was dipped vertically into the sol solution and held for $5 \mathrm{~min}$ until the sol-gel coating was formed on the $1.0 \mathrm{~cm}$ bare outer surface at the end of the wire. This process was repeated several times to obtain desired coating thickness. Then the fiber was dried at room temperature for $12 \mathrm{~h}$.

For the fibers prepared by immobilizing with epoxy-resin, the treated wire was firstly coated with a very thin and homogeneous layer of epoxy resin, and then inserted into MCF-1 (or Ph-MCF-1). The length of the wire covered by the powder was controlled as $2.0 \mathrm{~cm}$. Subsequently, the fiber was pulled out and air dried for $30 \mathrm{~s}$. This procedure was repeated several times until the prospective coating thickness was obtained.

\subsection{SPME procedures}

A volume of $10 \mathrm{~mL}$ of sample solution was placed in a $12 \mathrm{~mL}$ glass vial with a stir bar. The home-made fiber was immersed into the aqueous sample at $1000 \mathrm{rpm}$ stirring condition for $15 \mathrm{~min}$. After extraction, the fiber was removed and gently inserted into the SPME-HPLC interface to desorb analytes for $2 \mathrm{~min}$, then the eluted solution in desorption chamber was injected into the HPLC-UV for analysis.

\section{Results and discussion}

\subsection{FT-IR analysis of the MCF-1 and Ph-MCF-1}

FT-IR spectra (Fig. 1) was employed to verify the chemical anchorage of phenyl group for chemistry of the synthesis of

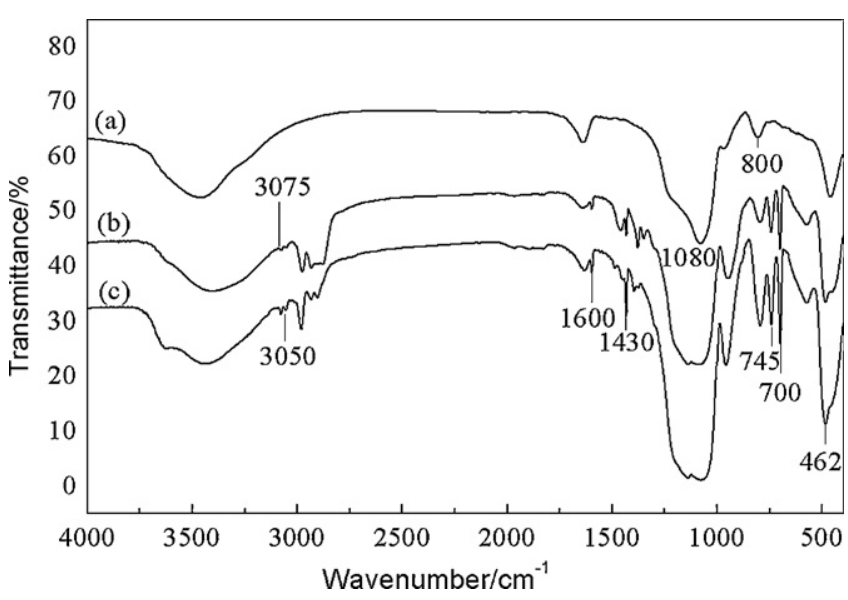

Fig. 1. FT-IR spectra of (a) MCF-1, (b) Ph-MCF-1 and (c) Ph-MCF-1A coating.

MCF-1 and its immobilization coating by sol-gel technology (MCF1A). Compared with MCF-1 (Fig. 1a), Ph-MCF-1 (Fig. 1b) and Ph-MCF-1A (Fig. 1c) all showed the typical phenyl group absorption peaks at $3075,3050,1600$ and $1430 \mathrm{~cm}^{-1}$. In particular, the peaks of single substituted phenyl group also appear at 745 and $700 \mathrm{~cm}^{-1}$, respectively. These data demonstrated that the phenyl groups have already been anchored into the framework MCFs materials and its coating.

\subsection{Characterization of MCF-1 and Ph-MCF-1}

To evaluate the microstructure of MCF-1 and Ph-MCF-1, Fig. 2 illustrates a representative TEM images. These micrographs indicate 3D interconnected uniform mesostructures with obvious ultralarge-pore structure because of the introduction of swelling agent, which is consistent with the pore structure parameters from nitrogen adsorption-desorption isotherms (Table 1 ). The structure of the honeycomb-like materials is in agreement with those of the MCFs materials previously reported [32,33]. These results indicate that the synthesized MCFs are promising materials for adsorption and separation applications in sample pretreatment.

\subsection{Morphological structure of MCF-1 and Ph-MCF-1 coatings}

Two types of fibers (MCF-1A and Ph-MCF-1A) were prepared by sol-gel method and the thickness of the sorbent coatings obtained from immobilization by epoxy-resin were measured as $185 \mu \mathrm{m}$ (Fig. 3a) and $195 \mu \mathrm{m}$ (Fig. 3b), respectively. No pores and channels appeared on the surface of coating. The coating thickness immobilized by epoxy-resin method fibers (MCF-1B and Ph-MCF-1B) was about $110 \mu \mathrm{m}$ (Fig. 4a and b) and $120 \mu \mathrm{m}$ (Fig. 4c and d), respectively. Comparing the sol-gel coating, the surface of fibers was very coarse and possessed porous structure.

\subsection{Optimization of SPME conditions}

In order to achieve the best extraction efficiency of the new coating (MCF-1A fiber as an example) for seven BFRs, several poten-

Table 1

Pore structure parameters of MCF-1 and Ph-MCF-1 materials.

\begin{tabular}{|c|c|c|c|c|c|}
\hline Material & BET surface area $\left(\mathrm{m}^{2} \mathrm{~g}^{-1}\right)$ & Pore volume $\left(\mathrm{cm}^{3} \mathrm{~g}^{-1}\right)$ & $D_{c}(\mathrm{~nm})^{\mathrm{a}}$ & $d_{100}(\mathrm{~nm})^{\mathrm{b}}$ & Wall thickness $(\mathrm{nm})^{\mathrm{c}}$ \\
\hline MCF-1 & 846.8 & 1.68 & 8.78 & 11.6 & 2.9 \\
\hline Ph-MCF-1 & 770.8 & 1.48 & 9.78 & 11.9 & 3.8 \\
\hline
\end{tabular}

a Cell diameter, $D_{c}$, determined from nitrogen adsorption-desorption.

b Determined from $2 d \sin \theta=n \lambda(n=1) . \theta$ and $\lambda$ determined from small-angle X-ray diffraction.

c Calculated by $a_{0}$ - pore size $\left(D_{c}\right)\left(a_{0}=2 d_{100} /\left(3^{1 / 2}\right)\right)$. 

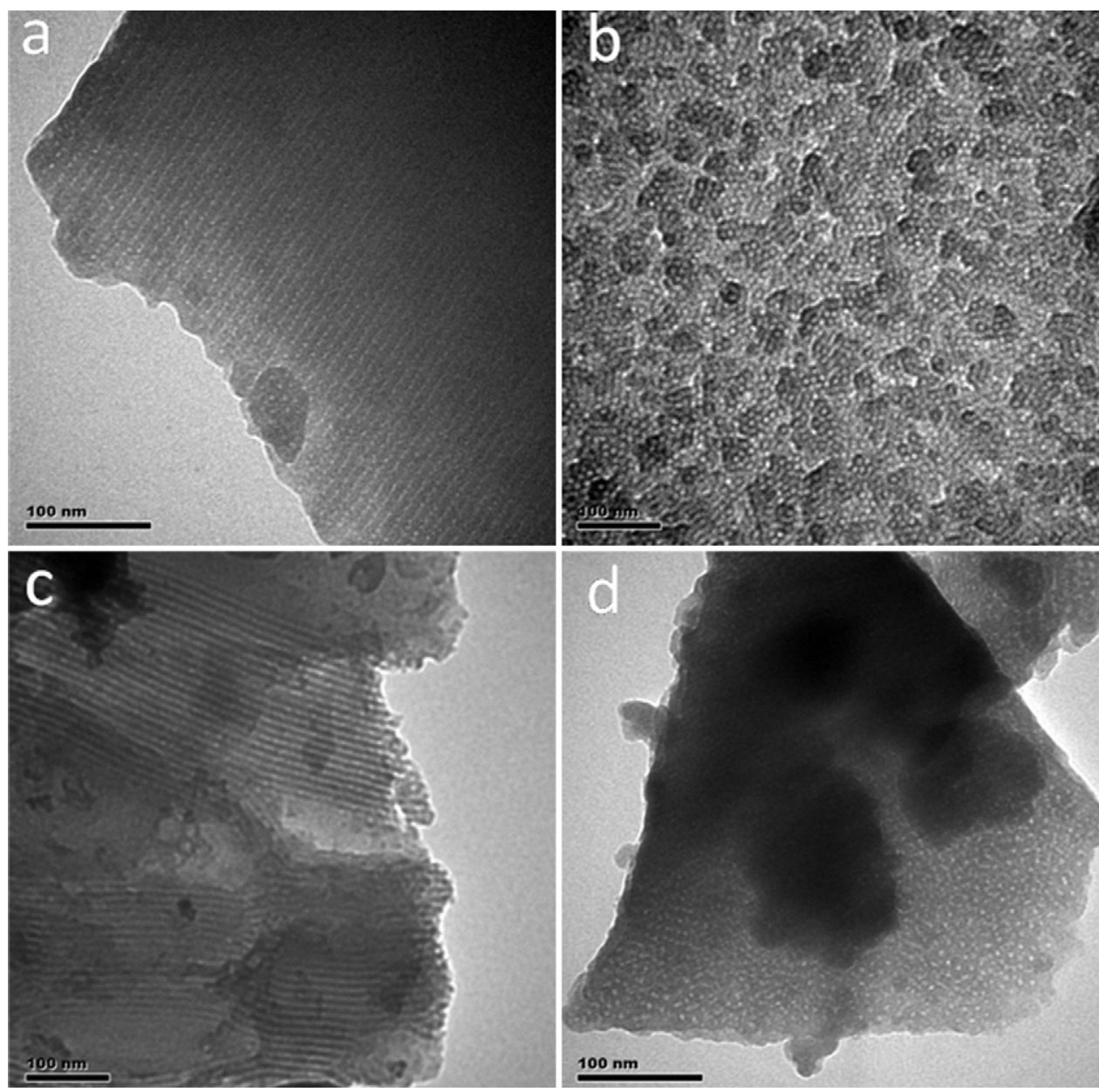

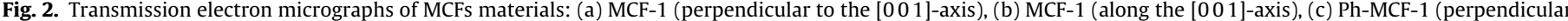
to the [ $\left[\begin{array}{lll}0 & 0 & 1\end{array}\right]$-axis) and (d) Ph-MCF-1 (along the [0 011$]$-axis).
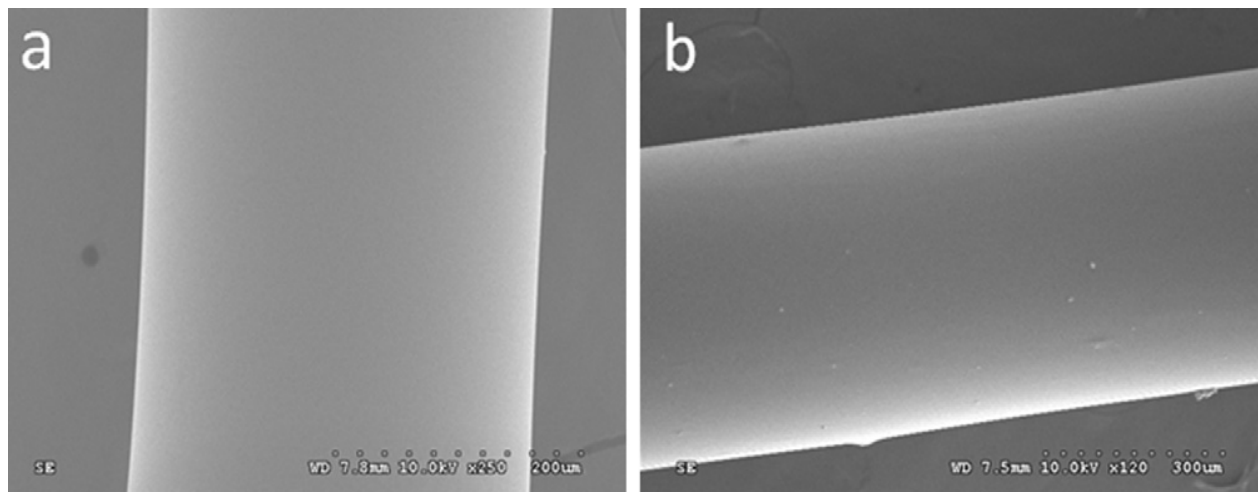

Fig. 3. SEM images of the fiber prepared by the sol-gel method: (a) MCF-1A (250x) and (b) Ph-MCF-1A (120x).

tial factors, including extraction temperature, extraction time, desorption time, stirring rate and ionic strength of samples were investigated and optimized. Optimization of these parameters was carried out on standard mixture solutions of BFRs with each target analytes at concentration of $50 \mu \mathrm{g} \mathrm{L}^{-1}$.

\subsubsection{Extraction temperature}

The extraction temperature is an important factor because of its potential influences on thermodynamics and kinetics of extraction equilibrium of analyte between fiber coating and solution.
Adsorption is generally an exothermic process, namely, the amount of adsorbed analytes decreases when temperature is increased. On the other hand, a higher temperature increases the diffusion rate of the analytes, leading to increase of the extraction rate. Extraction temperature profiles for BFRs ranging from 20 to $50{ }^{\circ} \mathrm{C}$ were investigated. As shown in Fig. 5a, the highest extraction of BPA and TBBPA was reached at $30^{\circ} \mathrm{C}$ and $40^{\circ} \mathrm{C}$, respectively, whereas the extraction efficiency of TBBPS-dbpe decreased along with increase in the temperature. For the other four analytes, the extraction temperature had no significant effect on the extraction efficiencies. Finally, 

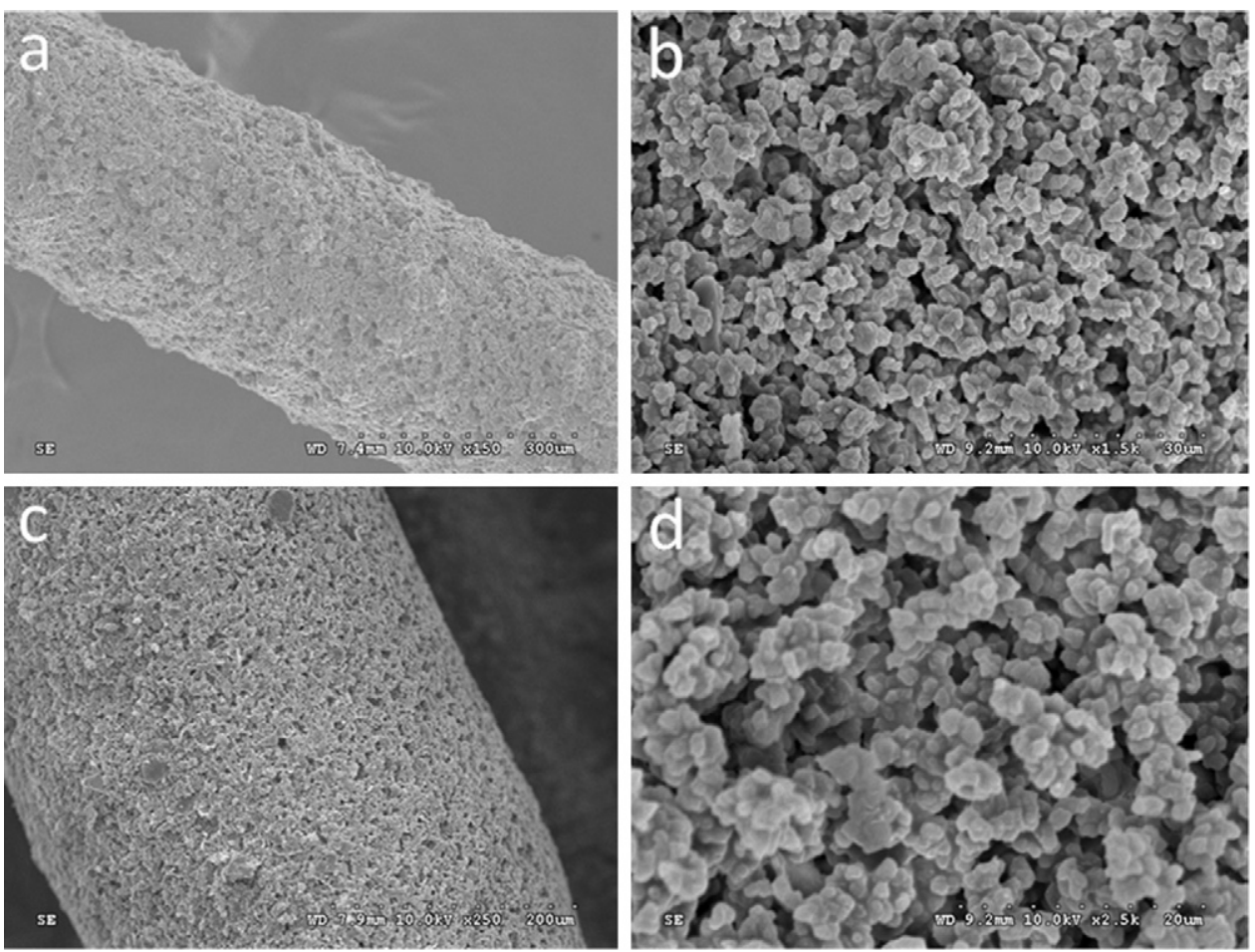

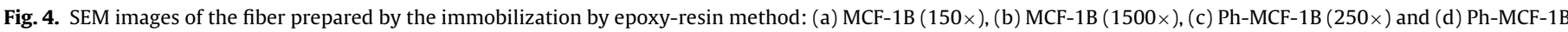
$(2500 \times)$.

considering the simplicity of operations and higher extraction efficiencies for all the seven BFRs, an extraction temperature of $30^{\circ} \mathrm{C}$ was chosen.

\subsubsection{Extraction time}

The effect of extraction time on the extraction efficiency was investigated from 10 to $60 \mathrm{~min}$. The amount of extracted analytes depends on the rate of mass transfer process at the interface of the samples phase and coating materials. The result showed that the responses of seven BFRs increased with the prolonged extraction time. It indicated that the equilibrium was not achieved after $60 \mathrm{~min}$ and the new coating fiber showed remarkable extraction capacity toward these analytes. On the other hand, the MCF-1A coating is a polymer-based solid extracting phase with macroporous structure. They extract analytes via adsorption rather than absorption (as PDMS), while adsorption is a competitive process and the highest extraction efficiency is not at the point of equilibrium. Therefore, an extraction time of $30 \mathrm{~min}$ was selected as a compromise working condition between time efficiency and method sensitivity.

\subsubsection{Desorption time}

The desorption time profiles ranging from 2 to $10 \mathrm{~min}$ were investigated. The peak height reached the maximum at $2 \mathrm{~min}$ for all the studied analytes, after 2 min, there were no significant differences of the peak height of these analytes. Therefore, the exposure time of $2 \mathrm{~min}$ in the SPME desorption chamber was used for the desorption of BFRs.

\subsubsection{Stirring rate}

In principle, extraction efficiency could be enhanced with the increase of stirring rate because it can accelerate mass transfer of the analytes between the aqueous sample and the SPME fiber. Stirring rate ranging from 600 to $1200 \mathrm{rpm}$ was investigated. The results indicated a positive effect on the peak height of BPA, TBBPA and TBBPA-dbpe but no significant effect on TBBPS and TBBPA-hee when stirring rate was less than $1000 \mathrm{rpm}$ (Fig. 5b). Further increase of stirring rate led to an obvious decrease in that of TBBPA-ae, TBBPS and TBBPS-dbpe, which was likely to the vigorous stirring resulted in magneton flutter and air bubble formation and then affect mass transfer. Consequently, the stirring rate of $1000 \mathrm{rpm}$ was selected in this work.
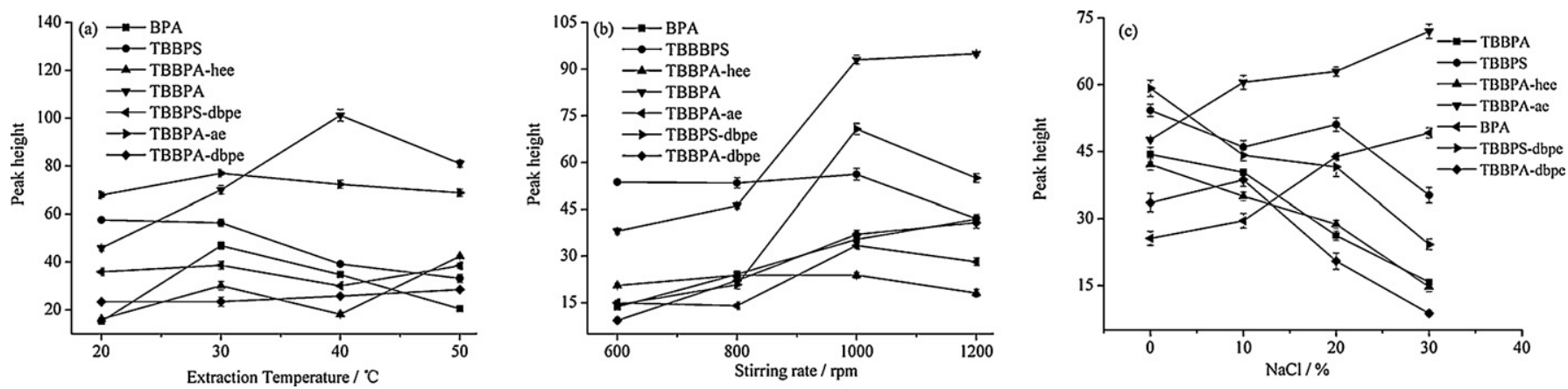

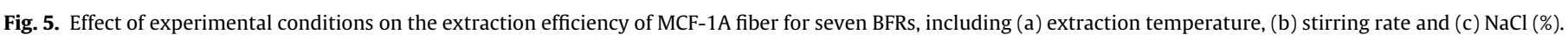




\subsubsection{Ionic strength of samples}

Addition of salt into aqueous samples can decrease the solubility of nonpolar organic compounds in water, which could increase the distribution coefficient of the analytes to the SPME fiber and thus enhance extraction efficiency. Nevertheless, it could increase the viscosity and density of the solution, and decrease the diffusion rate of the analytes, which is unfavorable to the analytes transferring to the fiber in direct SPME [11]. Here, the effect of ionic strength was determined by adding sodium chloride $(\mathrm{NaCl})(0-30 \%$ $m / v)$ into the sample solution. Fig. 5c shows that addition of $\mathrm{NaCl}$ had negative effect on extraction efficiencies of five analytes except BPA and TBBPA-ae, corroborating the results of other researchers $[6,16]$. Based on this, the salt was not added in this study. On the basis of the observations mentioned above, the conditions for the SPME of seven BFRs were determined as follows: extraction temperature, $30^{\circ} \mathrm{C}$; extraction time, $30 \mathrm{~min}$; desorption time, $2 \mathrm{~min}$; stirring rate, $1000 \mathrm{rpm}$.

\subsection{Comparison between the home-made and commercial fibers}

The seven semivolatile BFRs are benzenoid compound with medium polarity. To investigate the extraction ability of the four home-made fibers, commercial $100 \mu \mathrm{m}$ PDMS (nonpolar coating), $85 \mu \mathrm{m}$ PA (polar coating) and $65 \mu \mathrm{m}$ PDMS/DVB (middle-polar coating) were selected to perform SPME of seven BFRs under the same optimum conditions. From the results shown in Fig. 6, we could see that extraction efficiency of the four home-made fibers had superior extraction performance for BFRs than those of three commercial fibers. For the MCF-1A and Ph-MCF-1A fibers, their surface was smooth and similar to the commercial fibers, and thus resulted in the best reproducibility for each fiber. However, the thickness of home-made fibers were about 2-3-fold thicker than those of three commercial fibers; it is very crucial and beneficial for the absorption domination of these coatings. For MCF-1B and $\mathrm{Ph}-\mathrm{MCF}-1 \mathrm{~B}$ fibers, their rough surface and porous structure of the 3D network could provide larger surface area and thus improve extraction. All these indicated that MCFs material have a strong extraction ability to the seven BFRs.

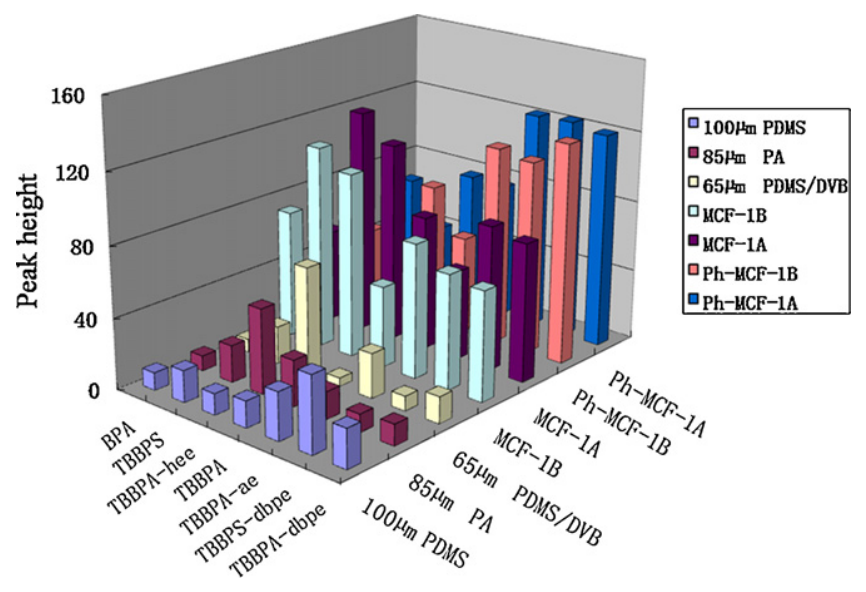

Fig. 6. Comparison between the home-made fibers and the commercial fibers under optimum conditions.

\subsection{Method validation}

To assess the extraction ability of the home-made fiber, MCF-1A fiber was selected for the subsequent experiments on target compounds analysis under optimum conditions. Table 2 summarizes the results of method validation. The linear range was tested from 5.0 to $1000 \mu \mathrm{g} \mathrm{L}^{-1}$ for each compound with exception of TBBPS from 1.0 to $1000 \mu \mathrm{g} \mathrm{L}^{-1}$. The linear correlation coefficient $\left(r^{2}\right)$ of seven analytes was from 0.9993 to 0.9999 in the tested range. The limits of detection (LODs) $(\mathrm{S} / \mathrm{N}=3)$ of the developed method were in the range of $0.4-0.9 \mu \mathrm{g} \mathrm{L}^{-1}$. The relative standard deviation (RSD) for five replicate extractions of analytes at $50 \mu \mathrm{g} \mathrm{L}^{-1}$ using same SPME fiber (MCF-1A) was $1.2-5.1 \%$. The reproducibility of fiber-to-fiber for five parallel prepared fibers for SPME of the BFRs was 2.5-6.5\% (RSD) and batch-to-batch was 3.2-6.7\% (RSD). The durability experiment for one fiber showed no significant change in the extraction efficiency over 150 cycles of SPME.

Table 2

Detection limits, linear range and repeatability of the proposed method.

\begin{tabular}{|c|c|c|c|c|c|c|}
\hline \multirow[t]{2}{*}{ Analyte } & \multirow[t]{2}{*}{ Linear range $\left(\mu \mathrm{g} \mathrm{L}^{-1}\right)$} & \multirow[t]{2}{*}{ Coefficient of correlation, $r^{2}$} & \multirow[t]{2}{*}{$\operatorname{LOD}\left(\mathrm{S} / \mathrm{N}=3, \mu \mathrm{g} \mathrm{L}^{-1}\right)$} & \multirow[t]{2}{*}{ Precision $^{\mathrm{a}}(\mathrm{RSD} \%, n=5)$} & \multicolumn{2}{|c|}{ Repeatability (RSD\%, $n=5)^{\mathrm{b}}$} \\
\hline & & & & & Fiber-to-fiber & Batch-to-batch \\
\hline BPA & $5.0-1000$ & 0.9997 & 0.7 & 3.6 & 3.8 & 5.8 \\
\hline TBBPS & $1.0-1000$ & 0.9997 & 0.4 & 4.6 & 6.5 & 6.0 \\
\hline TBBPA-hee & $5.0-1000$ & 0.9993 & 0.9 & 1.2 & 5.7 & 3.2 \\
\hline TBBPA & $5.0-1000$ & 0.9996 & 0.9 & 5.1 & 2.5 & 4.9 \\
\hline TBBPS-dbpe & $5.0-1000$ & 0.9999 & 0.8 & 1.2 & 4.4 & 6.7 \\
\hline TBBPA-ae & $5.0-1000$ & 0.9995 & 0.8 & 3.1 & 3.9 & 4.4 \\
\hline TBBPA-dbpe & $5.0-1000$ & 0.9993 & 0.8 & 3.3 & 3.2 & 5.6 \\
\hline
\end{tabular}

a Repeatability of one fiber (MCF-1A).

b The concentration of the standard solution was $50 \mu \mathrm{g} \mathrm{L}^{-1}$ for each compound, and other conditions were the optimized conditions.

Table 3

Determination of residues and recoveries of BFRs in real water samples using MCF-1A fiber.

\begin{tabular}{|c|c|c|c|c|c|c|c|c|}
\hline \multirow[t]{2}{*}{ Analyte } & \multicolumn{4}{|l|}{ Xiaoqing River } & \multicolumn{4}{|c|}{ Waste water of plant } \\
\hline & Original $\left(\mu \mathrm{g} \mathrm{L}^{-1}\right)$ & Found $\left(\mu g \mathrm{~L}^{-1}\right)$ & Recovery $^{\mathrm{a}}(\%)$ & $\mathrm{RSD}(\%, n=5)$ & Original $\left(\mu \mathrm{g} \mathrm{L}^{-1}\right)$ & Found $\left(\mu \mathrm{g} \mathrm{L}^{-1}\right)$ & Recovery $^{\mathrm{a}}(\%)$ & $\operatorname{RSD}(\%, n=5)$ \\
\hline BPA & 4.4 & 47.4 & 87.1 & 5.0 & 14.4 & 60.5 & 93.9 & 4.9 \\
\hline TBBPS & n.d. ${ }^{b}$ & 51.7 & 103.4 & 4.6 & 12.1 & 53.7 & 86.5 & 5.8 \\
\hline TBBPA-hee & n.d. & 47.3 & 94.6 & 4.3 & n.d. & 45.5 & 91.0 & 6.7 \\
\hline TBBPA & n.d. & 51.8 & 103.6 & 3.6 & 19.3 & 71.5 & 103.2 & 6.5 \\
\hline TBBPS-dbpe & n.d. & 45.7 & 91.4 & 5.5 & 9.5 & 52.0 & 87.4 & 5.6 \\
\hline TBBPA-ae & n.d. & 44.2 & 88.4 & 4.1 & 7.9 & 58.7 & 101.4 & 6.8 \\
\hline TBBPA-dbpe & n.d. & 51.7 & 103.4 & 5.8 & n.d. & 45.8 & 91.6 & 6.3 \\
\hline
\end{tabular}

a Spiked concentration of each compound was $50 \mu \mathrm{g} \mathrm{L}^{-1}$.

b Not detected. 


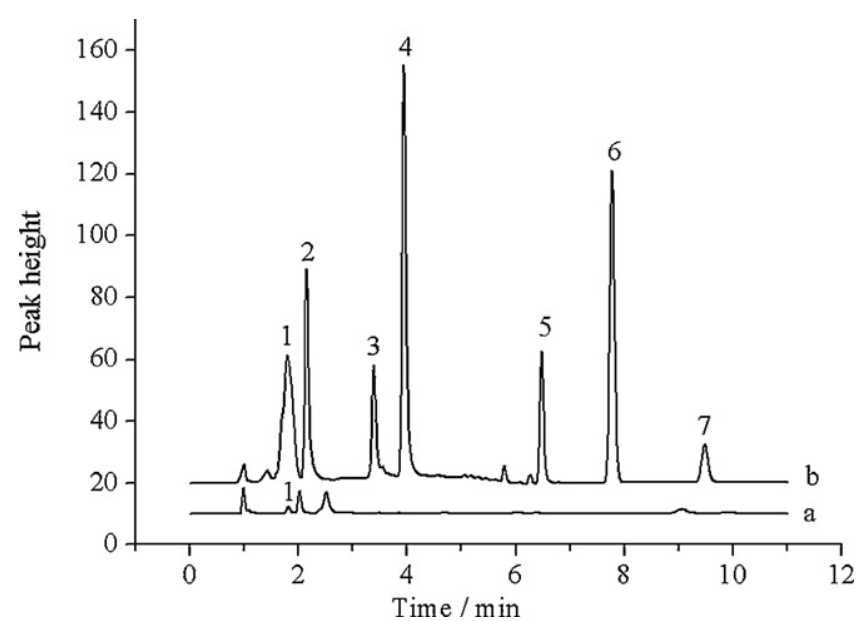

Fig. 7. The chromatogram of 7 BFRs obtained using the developed method in (a) the original Xiaoqing River samples and (b) the spiked Xiaoqing River samples (50 $\mathrm{g} \mathrm{L}^{-1}$ for each BFR were added) using MCF-1A fiber. (1) BPA, (2) TBBPS, (3) TBBPA-hee, (4) TBBPA, (5) TBBPS-dbpe, (6) TBBPA-ae and (7) TBBPA-dbpe. Experiment conditions: extraction temperature, $30^{\circ} \mathrm{C}$; extraction time, $30 \mathrm{~min}$; desorption time, $2 \mathrm{~min}$; stirring rate, $1000 \mathrm{rpm}$.

\subsection{Real samples analysis}

In order to test the applicability of the MCF-1A fiber, the method was applied to the analysis of the seven BFRs in real water samples, including samples from Xiaoqing River and treated wastewater from a plant which produce TBBPA and other brominated flame retardants (Shandong, China). The analytical results were given in Table 3. As a result, only BPA was found at $4.4 \mu \mathrm{g} \mathrm{L}^{-1}$ in Xiaoqing River, whereas BPA, TBBPS, TBBPA, TBBPS-dbpe and TBBPA-ae were found in wastewater with concentration range from 7.9 to $19.3 \mu \mathrm{g} \mathrm{L}^{-1}$. Spiking recoveries were determined to evaluate the accuracy with spiking concentrations of $50 \mu \mathrm{g} \mathrm{L}^{-1}$ for each compounds. Five replicate analysis were carried out and RSD was 3.4-6.8\%. The results showed good spiking recoveries, ranging from 86.5 to $103.6 \%$. Fig. 7 shows a typical chromatogram of Xiaoqing River samples with and without BFRs spiking obtained using the developed method for the MCF-1A fiber. The results demonstrated that the innovative SPME fiber has the reliability and applicability, and has high extraction efficiency and good selectivity for BFRs in this study.

\section{Conclusion}

In this paper, two kinds of home-synthesized MCFs materials, including MCF-1 and Ph-MCF-1, were firstly used as the coatings of SPME fibers, prepared by sol-gel and immobilization with epoxyresin methods, respectively. Compared with the commercial SPME fibers (PDMS, PA and CW/TPR), the home-made fibers exhibited higher extraction efficiency toward seven selected BFRs and many advantages, such as low cost, good reproducibility and long lifespan (more than 150 cycles). Under the optimum conditions, the home-made fibers were successfully applied to analyze BFRs in real water samples and achieved the satisfactory results with the recoveries of 86.5-103.6\%. The developed method exhibited good precision, a wide concentration range and offers a simple, rapid, sensitive and inexpensive pretreatment way for determination of trace BFRs. Therefore, future efforts should be made to enhance the resistance of home-made SPME fibers to high temperatures to better suit GC methods. Moreover, more complex matrices such as soil and biosolids should be tested to extend the environmental applications of this method.

\section{Acknowledgements}

This work was generously supported by the Natural Science Foundation of China (Nos. 20977096 and 21107086), National Key Water Program (No. 2009ZX07527-005), Key Lab of Bioelectrochemistry \& Environmental Analysis of Gansu Province and the Key Laboratory of Ecological Environment Related Polymer Materials of Ministry of Education.

\section{Appendix A. Supplementary data}

Supplementary data associated with this article can be found, in the online version, at http://dx.doi.org/10.1016/j.aca.2012.09.030.

\section{References}

[1] T. Hyotylainen, M.L. Riekkola, Anal. Chim. Acta 614 (2008) 27-37.

[2] J. Pawliszyn (Ed.), Handbook of Solid Phase Microextraction, Chemical Industry Press, Beijing, 2009.

[3] G. Ouyang, J. Pawliszyn, Anal. Chim. Acta 627 (2008) 184-197.

[4] S. Risticevic, H. Lord, T. Gorecki, C.L. Arthur, J. Pawliszyn, Nat. Protoc. 5 (2010) 122-139.

[5] J. Wang, D. Jiang, Z. Gu, X. Yan, J. Chromatogr. A 1137 (2006) 8-14.

[6] X. Hu, J. Pan, Y. Hu, Y. Huo, G. Li, J. Chromatogr. A 1188 (2008) 97-107.

[7] X. Cui, Z. Gu, D. Jiang, Y. Li, H. Wang, X. Yan, Anal. Chem. 81 (2009) 9771-9777.

[8] J.W. Zewe, J.K. Steach, S.V. Olesik, Anal. Chem. 82 (2010) 5341-5348.

[9] I. Minet, L. Hevesi, M. Azenha, J. Delhalle, Z. Mekhalif, J. Chromatogr. A 1217 (2010) 2758-2767.

[10] X.M. Wang, X.Z. Du, H.H. Rao, X.Q. Lu, J. Sep. Sci. 33 (2010) 3239-3244.

[11] J.G. Hou, Q. Ma, X.Z. Du, H.L. Deng, J.Z. Gao, Talanta 62 (2004) 241-246.

[12] D.H. Wang, J. Xing, J.G. Peng, C.Y. Wu, J. Chromatogr. A 1005 (2003) 1.

[13] A. Kumar, A.K. Gaurav, D.K. Malik, T.B. Singh, Anal. Chim. Acta 610 (2008) $1-12$.

[14] F.G. Tamayo, E. Turiel, A. Martin-Esteban, J. Chromatogr. A 1152 (2007) 32-40.

[15] Q. Li, X. Wang, D. Yuan, J. Chromatogr. A 1216 (2009) 1305-1311.

[16] H. Zhang, H.K. Lee, J. Chromatogr. A 1218 (2011) 4509-4516.

[17] S. Zhang, Z. Du, G. Li, Anal. Chem. 83 (2011) 7531-7541.

[18] G.Q. Lu, X.S. Zhao, Nanoporous Mater. Sci. Eng. 4 (2004) 897-905 (Series on chemical engineering).

[19] A. Taguchi, F. Schuth, Microporous Mesoporous Mater. 77 (2005) 1-45.

[20] M.J. Hudson, J.P. Knowles, P.J.F. Harris, D.B. Jackson, M.J. Chinn, J.L. Ward, Microporous Mesoporous Mater. 75 (2004) 121-128.

[21] C. Charnay, S. Bégu, C. Tourné-Péteilh, L. Nicole, D.A. Lerner, J.M. Devoiselle, Eur. J. Pharm. Biopharm. 57 (2004) 533-540.

[22] F. Qu, G. Zhu, S. Huang, S. Li, J. Sun, D. Zhang, S. Qiu, Microporous Mesoporous Mater. 92 (2006) 1-9.

[23] L.P. Mercuri, L.V. Carvalho, F.A. Lima, C. Quayle, M.C.A. Fantini, G.S. Tanaka, W.H Cabrera, M.F.D. Furtado, D.V. Tambourgi, J.R. Matos, M. Jaroniec, O.A. SantAnna, Small 2 (2) (2006) 254-256.

[24] P. Schmidt-Winkel, W.W. Lukens Jr., D. Zhao, P. Yang, B.F. Chmelka, G.D. Stucky, J. Am. Chem. Soc. 121 (1999) 254-255.

[25] K. De Witte, V. Meynen, M. Mertens, O.I. Lebedev, G. Van Tendeloo, A. Sepulveda-Escribano, F. Rodriguez-Reinoso, E.F. Vansant, P. Cool, Appl. Catal. B: Environ. 84 (1-2) (2008) 125-132.

[26] Y.M. Liu, W.L. Feng, L.C. Wang, Y. Cao, W.L. Dai, H.Y. He, K.N. Fan, Catal. Lett. 106 (3-4) (2006) 145-152.

[27] A. Nakajima, D. Saigusa, N. Tetsu, T. Yamakuni, Y. Tomioka, T. Hishinuma, Toxicol. Lett. 189 (2009) 78-83.

[28] A. Covaci, A.C. Gerecke, R.J. Law, S. Voorspoels, M. Kohler, N.V. Heeb, H. Leslie, C.R. Allchin, J. De Boer, Environ. Sci. Technol. 40 (2006) 3679-3688.

[29] G.F. Jurgella, A. Marwah, J.A. Malison, R. Peterson, T.P. Barry, Gen. Comp. Endocrinol. 148 (2006) 273-281.

[30] R.J. Law, P. Bersuder, C.R. Allchin, J. Barry, Environ. Sci. Technol. 40 (2006) 2177-2183.

[31] X.M. Wang, X.Z. Du, C.L. Li, X. Cao, Appl. Surf. Sci. 254 (2008) 3753-3757.

[32] F. Carn, A. Colin, M.F. Achard, H. Deleuze, E. Sellier, M. Birot, R. Backov, J. Mater. Chem. 14 (2004) 1370-1376.

[33] Q. Li, Z.X. Wu, D. Feng, B. Tu, D.Y. Zhao, J. Phys. Chem. C 114 (2010) 5012-5019. 